\title{
EXPEDIENTE
}

\section{EDITOR CIENTÍIFICO}

Celia Linhares, UFRRJ/PPGEDUC, Brasil

\section{EDITORES GERENTES}

Adrianne Ogêda Guedes, UNIRIO, Brasil

Márcia Denise Pletsch, UFRRJ - PPGEDUC, Brasil

Rosimeri de Oliveira Dias, FFP/UERJ, Brasil

\section{COMISSÃO EDITORIAL}

Adrianne Ogêda Guedes, UNIRIO, Brasil

Carmen Sanches, UNIRIO, Brasil

Denize Sepúlveda, FFP/UERJ, Brasil

Jonas Alves da Silva Jr., UFRRJ/IM, Brasil

Léa Velocina Vargas Tiriba, UNIRIO, Brasil

Marcia Denise Pletsch, UFRRJ/IM, Brasil

Rosimeri de Oliveira Dias, FFP/UERJ, Brasil

Vania Finholdt Leite, FFP/UERJ, Brasil

\section{EDITOR DOSSIÊ CORPO EM MOVIMENTO}

Adrianne Ogêda Guedes, UNIRIO, Brasil

Lea Tiriba, UNIRIO, Brasil.

Lívia Lage, UNIRIO, Brasil

\section{CONSELHO EDITORIAL}

Prof $^{a} \operatorname{Dr}^{\mathrm{a}}$ Ana Maria Monteiro, UFRJ, Brasil

Prof. Dr. Carlos Eduardo Ferraço, Professor Associado da UFES, Brasil

Prof. Dr. Carlos Skliar, FLACSO-Argentina, Brasil

Prof $^{a}$ Dr $^{a}$ Catherine Walsh, Profesora de la Universidad Andina Simón Bolivar/Equador, Equador

Prof ${ }^{a} \operatorname{Dr}^{\mathrm{a}}$ Filomena Maria de Arruda Monteiro, Professora Associada da UFMT, Brasil

Prof. Dr. Guilherme do Val Toledo Prado, UNICAMP, Brasil

Prof $^{a} \operatorname{Dr}^{a}$ Inês Assunção de Castro Teixeira, Professora Associada da UFMG, Brasil

$\operatorname{Prof}^{\mathrm{a}} \mathrm{Dr}^{\mathrm{a}}$ Maura Corcini Lopes

Prof $^{a} \operatorname{Dr}^{\mathrm{a}}$ Maria Teresa Esteban, Professora Associada da UFF, Brasil

Prof. Dr. Marcos Reigotta, Professor Titular da UNISO, Brasil

Prof Nelson De Luca Pretto, Professor Titular da Faculdade de Educação da

Universidade Federal da Bahia, Brasil

Prof $^{\mathrm{a}} \mathrm{Dr}^{\mathrm{a}}$ Nilma Lino Gomes, Professora Adjunta da UFMG, Brasil

Prof $^{a}$ Dr $^{a}$ Virginia de Oliveira Silva, Professora Adjunto da UFPB, Brasil 


\section{REVISORES}

Bruna Fonseca Teixeira

Jeane de Araújo Silva

Telma da Graça de Lima Lage

Márcio Mori

EDITORES DE APOIO

Cecilia Nahra

Izadora Martins da Silva de Souza

CAPA

Guilherme Gonçalves

FOTOGRAFIA DA CAPA

Cecília Figueiredo 